\title{
Fluctuación diaria del fitoplancton en la capa superficial del océano durante la primavera de 1997 en el norte de Chile (20 $\left.18^{\circ} \mathrm{S}\right)$ : I. Biomasa Pigmentaria
}

\author{
Daily fluctuation of phytoplankton in the upper layer of the ocean \\ during the spring of 1997 in the north of Chile (20¹8’ S): \\ I. Pigment biomass \\ Edgardo Santander, Liliana Herrera y Jessica Pizarro \\ Universidad Arturo Prat , Departamento de Ciencias del Mar \\ Casilla 121, Iquique, Chile. \\ esantan@cec.unap.cl
}

\begin{abstract}
Resumen.- Se analiza la respuesta de la biomasa fitoplanctónica, sobre una escala diaria, a los cambios en la estructura física y química producidos por un evento de surgencia. Los resultados revelan la existencia de un acoplamiento entre las variables físicas y químicas, y una alta variabilidad espacial y temporal en la concentración de clorofila-a. Esta última se caracterizó por presentar un desfase de dos a tres días en su respuesta a condiciones favorables, observándose un cambio en las fracciones fitoplanctónicas responsables de las mayores biomasas.

El periodo de estudio coincidió con la presencia de "El Niño" 1997-1998, pero los resultados no reflejaron la condición anómala asociada a dicho evento.
\end{abstract}

Palabras claves: Biomasa pigmentaria, surgencia, ambiente físico, ambiente químico, ENOS.

\begin{abstract}
The response of phytoplanktonic biomass to the changes in the physical and chemical structure produced by an upwelling event is analyzed in a daily scale. The results show a physical-chemical coupling, and a high spatial and temporal variability in the chlorophyll concentration. The response of chlorophyll was characterized by a two or three days lag to favorable conditions, and a change in the phytoplanktonic fractions responsible of the most important concentration was observed.

The study period coincided with "El Niño” 1997-1998 presence, but the results do not show anomaly conditions associated with this event.
\end{abstract}

Keywords: Pigmentary biomass, upwelling, physics and chemical environment, ENSO.

\section{Introducción}

Los procesos de surgencia costera en la zona norte de Chile, particularmente frente a Iquique, son permanentes con una mayor intensidad hacia períodos cálidos (primavera y verano), en contraste a períodos fríos (otoño e invierno), lo cual depende de factores locales como topografía de fondo y puntas y cabos (Fuenzalida 1992). El análisis diario de este índice ha demostrado que los eventos de surgencia se presentan con una frecuencia de 5 a 7 días con períodos de relajación de 3 (Fuenzalida 1990).

Es ampliamente reconocido que el estrés que el viento ejerce sobre la superficie del océano introduce energía en la dimensión vertical y horizontal que puede afectar a la producción planctónica, ya sea por turbulencia que genera fluctuaciones en la capa de mezcla, como por la transformación de la capa superficial a consecuencia del movimiento costa afuera de esta masa (Boje \& Tomkzac 1978, Barber \& Smith 1981, Arcos et al. 1987, Wrobleski \& Richman 1987, Bernal 1990)

El fitoplancton en esta misma región presenta una comunidad acoplada a la estructura física con al menos dos grupos principales de fitoplancteres. Uno asociado a “aguas de surgencias”, y otro a aguas relativamente más cálidas. Su abundancia y biomasa sigue una tendencia similar con mayores concentraciones en sectores influenciados por surgencia, en contraste a zonas con 
aguas más cálidas con diferencias de al menos un orden de magnitud (Herrera 1990, Santander 1993).

Desde el punto de vista de la biomasa fitoplanctónica (biomasa pigmentaria), la concentración de clorofila "a" es el estimador químico más utilizado en investigaciones marinas (Billington 1991). Ahumada et al. (1991) han señalado que la distribución de la biomasa fitoplanctónica está relacionada con el enriquecimiento de nutrientes durante un evento de surgencia, indicando que su carga favorece el crecimiento del fitoplancton, a lo cual debe sumarse la estabilidad de la columna de agua. En este sentido, Legendre \& Demers (1984) señalan que la alternancia entre períodos de estabilidad e inestabilidad de la columna de agua incrementan la producción primaria.

Se debe recordar que el enlace entre las variables físicas y biológicas se estructura en espacio y tiempo (Haury et al. 1978), así cada propiedad física y biológica puede variar dentro de un estrecho rango espacio-temporal, el punto relevante es que diferentes patrones emergen a partir de diferentes escalas de investigación (Wiens 1989, Harris 1980). Al respecto, las observaciones de biomasa fitoplanctónica en el Norte de Chile han abarcado grandes extensiones espaciales del océano y en escalas estacionales (Arcos \& Salamanca 1984, Ahumada et al. 1991, Marin \& Olivares 1999), lo cual se ha traducido en escasa información acerca del comportamiento de la biomasa pigmentaria en escalas temporales diarias, y en particular a través de un evento de surgencia. Cabe destacar que cada evento de surgencia conduce a cambios en las propiedades físicas y químicas locales de la capa superficial del océano, lo cual modularía la distribución y magnitud de la biomasa del fitoplancton. El objetivo de este estudio es analizar la variabilidad de alta frecuencia de algunos factores relevantes a la ecología del fitoplancton, e inferir el posible control hidrodinámico que se ejerce sobre la biomasa fitoplanctónica, por medio de variables físicas y químicas.

\section{Materiales y Métodos}

Esta investigación fue llevado a cabo en la zona costera de Iquique (20¹8'S), donde se establecieron 6 estaciones paralelas a la línea de costa, con una separación latitudinal de $1 \mathrm{mn}$ y longitudinal de $2 \mathrm{mn}$ (Fig. 1). Los muestreos se llevaron a cabo desde el 26 de agosto al 17 de septiembre, lo cual se corresponde con el comienzo del "bloom" primaveral del fitoplancton (Alvial \& Avaria 1982).

\section{Longitud $\left({ }^{\circ} \mathrm{W}\right)$}

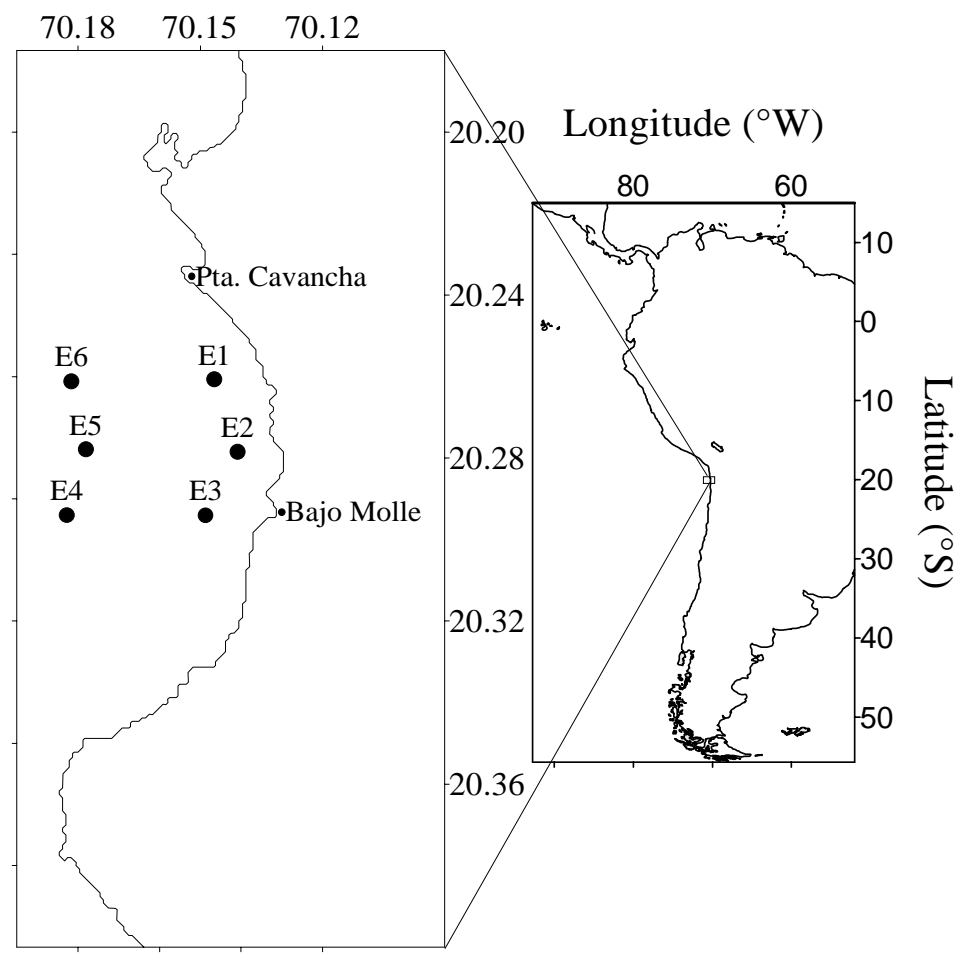

Figura 1

Area de estudio y posición de las estaciones.

Sampling area and geographical position of the stations. 
El índice de surgencia se estimó a partir de la velocidad del viento de acuerdo a Bakun (1973), con datos tomados en el aeropuerto Diego Aracena. La temperatura, salinidad y densidad se midieron a través de un CTD SeaBird 19. El análisis del oxígeno disuelto fue realizado según la modificación de Carpenter (1965) para el método de Winkler (Parsons et al. 1984). La penetración de la luz se midió mediante el uso del disco Secchi. La concentración de nitratos, nitritos y fosfatos se midió según las técnicas descritas en Parsons op. cit.. La Clorofila "a" se determinó mediante la técnica fluorométrica extractiva (acetona al 90\%), utilizando filtros GF/F (Whatmann), de acuerdo a lo propuesto por Parsons op. cit. El recuento de fitoplancton se hizo según el método del microscopio invertido de Utermöhl (1958). Para el análisis estadístico se realizaron correlaciones de rangos de Spearman (Zar 1984).

\section{Resultados}

Eventos de surgencia: La fluctuación del índice de surgencia (IS) horario (Fig. 2) evidenció condiciones siempre favorables para la generación de estos procesos (Fig. 2), destacándose que durante el período de estudio, hubo un claro predominio de los vientos S y SW (Fig. 3A), caracterizado por velocidades entre 4 y $7 \mathrm{~m} / \mathrm{s}$ (Fig. 3 A у B).

La señal del IS indica que los muestreos fueron iniciados después de la ocurrencia de un fuerte evento caracterizado por una persistencia aproximada de 7 días, seguido de una fase de relajación de tres. A partir del 24 de agosto el IS muestra un nuevo pulso caracterizado por la ocurrencia de cuatro eventos acoplados de intensidad moderada a baja. Hacia el final de la serie se observa un ultimo evento de mayor magnitud.

Condiciones oceanográficas:La estructura termal mostró una condición relativamente cálida especialmente en la capa más superficial, la cual desaparece el día 4 de septiembre (Fig. 4A y 5A). Posteriormente se detectó un enfriamiento hasta el 11 de septiembre. Tal período es coincidente con el proceso intrusivo de aguas subsuperficiales, denotado claramente por el ascenso de la isoterma de $17^{\circ} \mathrm{C}$ (Fig. 4A y 5A). A contar de esta misma fecha, se observó la aparición y profundización de la isoterma de $18^{\circ} \mathrm{C}$, llamando la atención que este evento fue mucho más evidente en las estaciones costeras. Hacia el final del período de estudio se produce un nuevo enfriamiento (Fig. 4A), especialmente en las estaciones costeras (Fig. 6A). Las diferencias entre estos sectores fueron en promedio del orden de $0,6^{\circ} \mathrm{C}$.

Por su parte, la estructura halina mostró un marcado gradiente a partir del comienzo del estudio, con valores que fluctuaron entre 35,0 y 35,3 psu, para luego observar un ascenso de aguas de carácter menos salino en la forma de un continuo. Las figuras $4 \mathrm{~B}$ y $5 \mathrm{~B}$ muestran claramente la intrusión de aguas menos salinas coincidente con el tercer pulso de los eventos de viento acoplados (Fig. 5B). De igual forma durante el calentamiento de las aguas superficiales se detectaron cambios visualizados por la profundización de la isohalina de 35 psu, lo cual fue más marcado en las estaciones oceánicas que en las costeras. El campo de densidades muestra el mismo patrón que el observado para temperatura, incluida la aparición de aguas menos densas durante los días 12 y 14 de septiembre (Figuras 4C y 5C).

Estructura Química: El ambiente químico mostró una buena coherencia con los patrones oceanográficos antes descritos, así, el oxígeno disuelto mostró claramente la entrada de aguas subsuperficiales, con concentraciones menores a 3,0 mL/L denotado por la oxilínea de 5. Llama la atención que esta isolínea coincide con la evolución de la isoterma de $18^{\circ} \mathrm{C}$, denotando un acoplamiento con la estructura física (Figuras 4D y 5D).

En el caso de los nitratos, estos presentaron concentraciones menores en los primeros metros de la columna de agua $(<9,0 \mu \mathrm{M})$, donde altas temperaturas coincidieron con bajas concentraciones de esta sal. En tal sentido, y coincidente con el ascenso de aguas más frías, se detectaron mayores niveles de nitrato en las capas más profundas (> $13 \mu \mathrm{M})$ (Fig. 6A y 7A). Se observó un patrón diferente entre las estaciones costeras y oceánicas. Estas diferencias se hicieron más clara a partir del 5 de septiembre.

El fosfato se caracterizó por presentar mayores concentraciones al inicio del período de estudio, con una capa superficial homogénea. Por un lado, en las estaciones costeras, concentraciones superiores a $3 \mu \mathrm{M}$, se detectaron desde el inicio hasta el 11 de septiembre, a partir del cual se observó una disminución en sus concentraciones (Figuras 6B y 7B), condición que se mantuvo hasta el final del período de estudio. Por otro, las estaciones más oceánicas presentaron mayores fluctuaciones destacando que concentraciones superiores a 3,0 $\mu \mathrm{M}$, solo se observaron entre los días 26 y 31 de agosto. Entre el 1 y el 3 de septiembre se apreciaron concentraciones menores a 3,0 $\mu \mathrm{M}$.

Claramente los patrones de fluctuación en las concentraciones de estos dos nutrientes fueron diferentes. Por un lado, el fósforo presentó un patrón simple y consistente que el observado para el nitrato, sin embargo, convergen es en que sus los niveles nunca se observaron realmente bajos, lo cual nos sugiere que durante el desarrollo de este estudio ninguno se encontró en concentraciones limitantes (en el sentido del mínimo de Liebeg). Al respecto, se exploró en la “razón” de Redfield. La figura 8 muestra que el sistema mostró un fuerte desbalance, debido principalmente a una menor carga de nitrato. De manera que sería el nitrato 
quien potencialmente limitaría a los productores primarios en la zona de estudio.

Biomasa fitoplanctónica: La clorofila-a se caracterizó por presentar altas concentraciones durante la mayor parte del período de estudio ( $>1,0 \mu \mathrm{g} / \mathrm{L}$ ). Desde el comienzo de los muestreos y hasta el 1 de septiembre se detectaron concentraciones superiores a $3 \mu \mathrm{g} / \mathrm{L}$, patrón que fue observado en las seis estaciones analizadas (Figuras 5C y 7C). A partir de esta fecha las concentraciones de clorofila descendieron bajo los 2,0 $\mu \mathrm{g} / \mathrm{L}$.

En las estaciones costeras se detectó la formación de un parche subsuperficial el cual alcanzó su pleno desarrollo el día 12 de septiembre, donde se detectaron las más altas concentraciones (> 11,0 $\mu \mathrm{g} / \mathrm{L}$ ). Posteriormente, sólo se detectó en el nivel más superficial concentraciones mayores a $5 \mu \mathrm{g} / \mathrm{L}$ (Fig. 5C). En el caso de las estaciones más oceánicas éstas presentaron niveles entre 2 y $3 \mu \mathrm{g} / \mathrm{L}$ (Fig. 7C), y sólo hacia el final del estudio se observaron concentraciones superiores a $5 \mu \mathrm{g} / \mathrm{L}$.

Para el caso de la concentración de células fitoplanctónicas, solo se dispuso de información para las estaciones costeras (Fig. 5D). Los recuentos celulares presentan un comportamiento similar al observado para la clorofila sobre todo al comienzo del período de estudio, donde concentraciones superiores 2*105 cél/L predominaron, seguido de una disminución hasta niveles cercanos a 4*104 cél/L. Hacia el final del estudio se detectaron concentraciones mayores a 2*105 cél/L. Llama la atención la ausencia de un máximo en la concentración de células para el intervalo donde se detectaron las máximas concentraciones de clorofila-a.

Análisis de correlación: Las tablas 1 y 2 resumen los resultados del análisis de correlación de rangos. De estas tablas se puede observar que la temperatura y salinidad siempre se relacionaron significativamente, lo cual revela que las condiciones oceanográficas respondieron a un solo proceso forzante, que en este caso fue la surgencia costera. También se puede visualizar que el campo químico presentó coherencia con las condiciones oceanográficas, lo cual puede ser deducido de las correlaciones significativas entre la salinidad y el contenido de oxígeno, lo cual a su vez, nos permite inferir que las concentraciones de oxígeno serían consecuencia de procesos físicos más que biológicos. Este acoplo también se ve refrendado por las correlaciones significativas entre el nitrato $\mathrm{y}$ la salinidad ( $\mathrm{p}<0,05)$. En cambio, la concentración de clorofila se caracterizó por no presentar correlación significativa con ninguna de las demás variables analizadas.

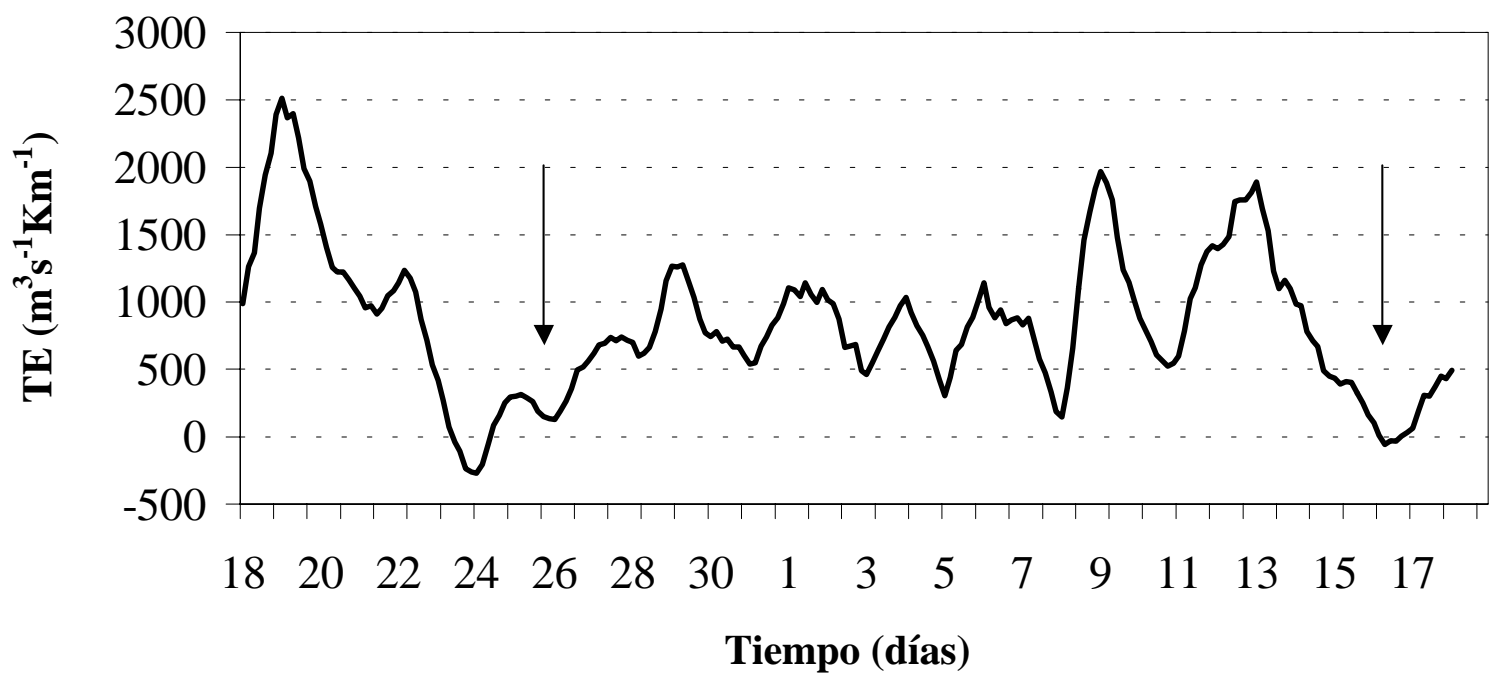

Figura 2

Promedio diario de un índice de surgencia para Iquique, durante el período comprendido entre el 17 de agosto y el 20 de septiembre de 1997.

Daily mean upwelling index to Iquique, during the period between August 17 and September 20 of 1997. 

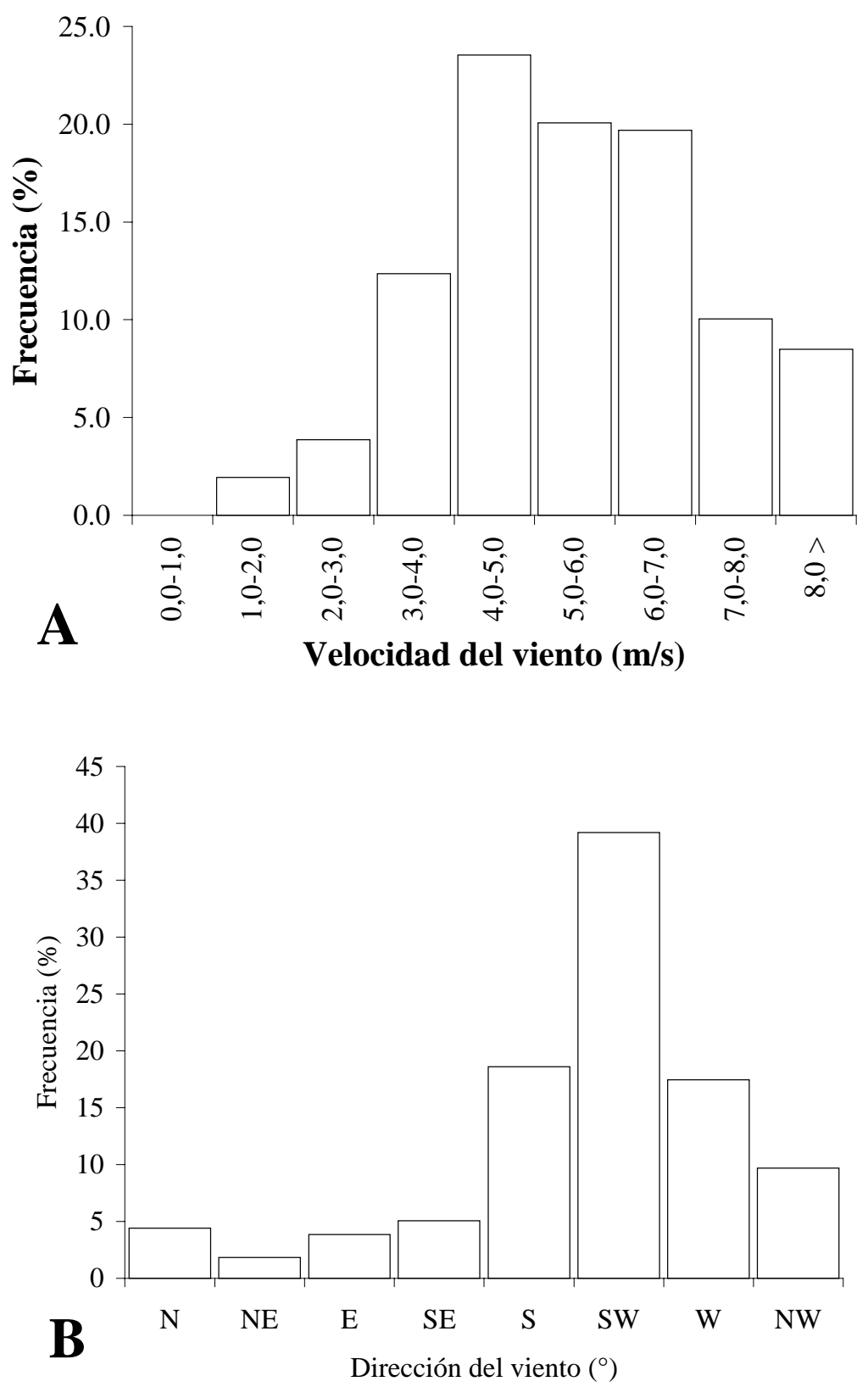

Figura 3

Frecuencia en los vientos para la componente paralela a la costa (A), y frecuencia en la dirección del viento para la componente paralela a la costa $(B)$.

Frequency in the winds for the parallel component to the coast (A), and frequency in the directions of the wind (B). 

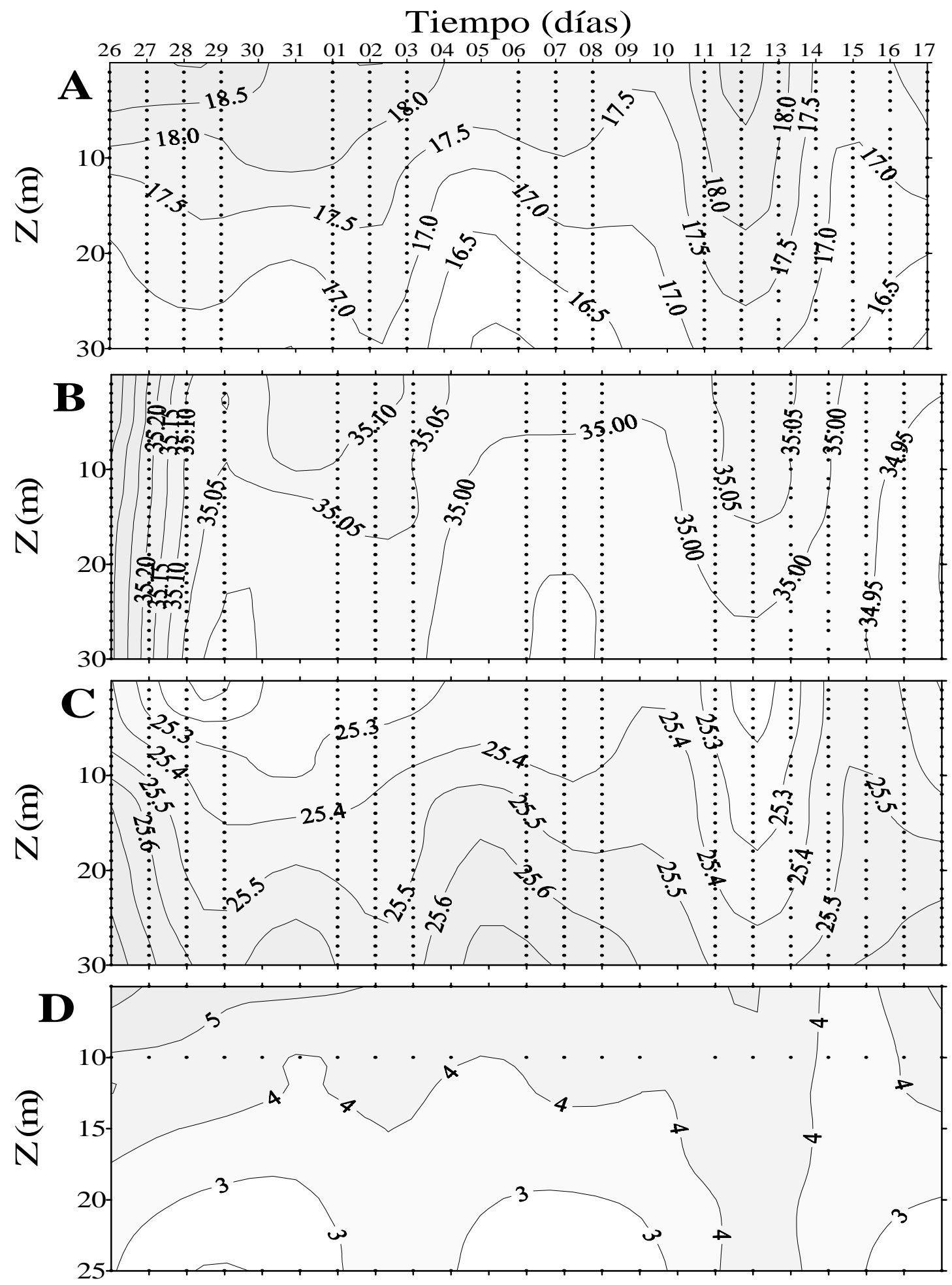

Figura 4

Distribución vertical para la estación 3 de (A) temperatura $\left({ }^{\circ} \mathrm{C}\right)$, (B) salinidad (psu), (C) densidad (unidades de sigma-t), y (D) oxígeno disuelto $(\mathrm{mL} / \mathrm{L})$.

Vertical distribution for the station 3 of (A) temperature $\left({ }^{\circ} \mathrm{C}\right.$ ), (B) salinity (psu), (C) density (sigma-t units), and (D) dissolved oxygen $(\mathrm{mL} / \mathrm{L})$. 

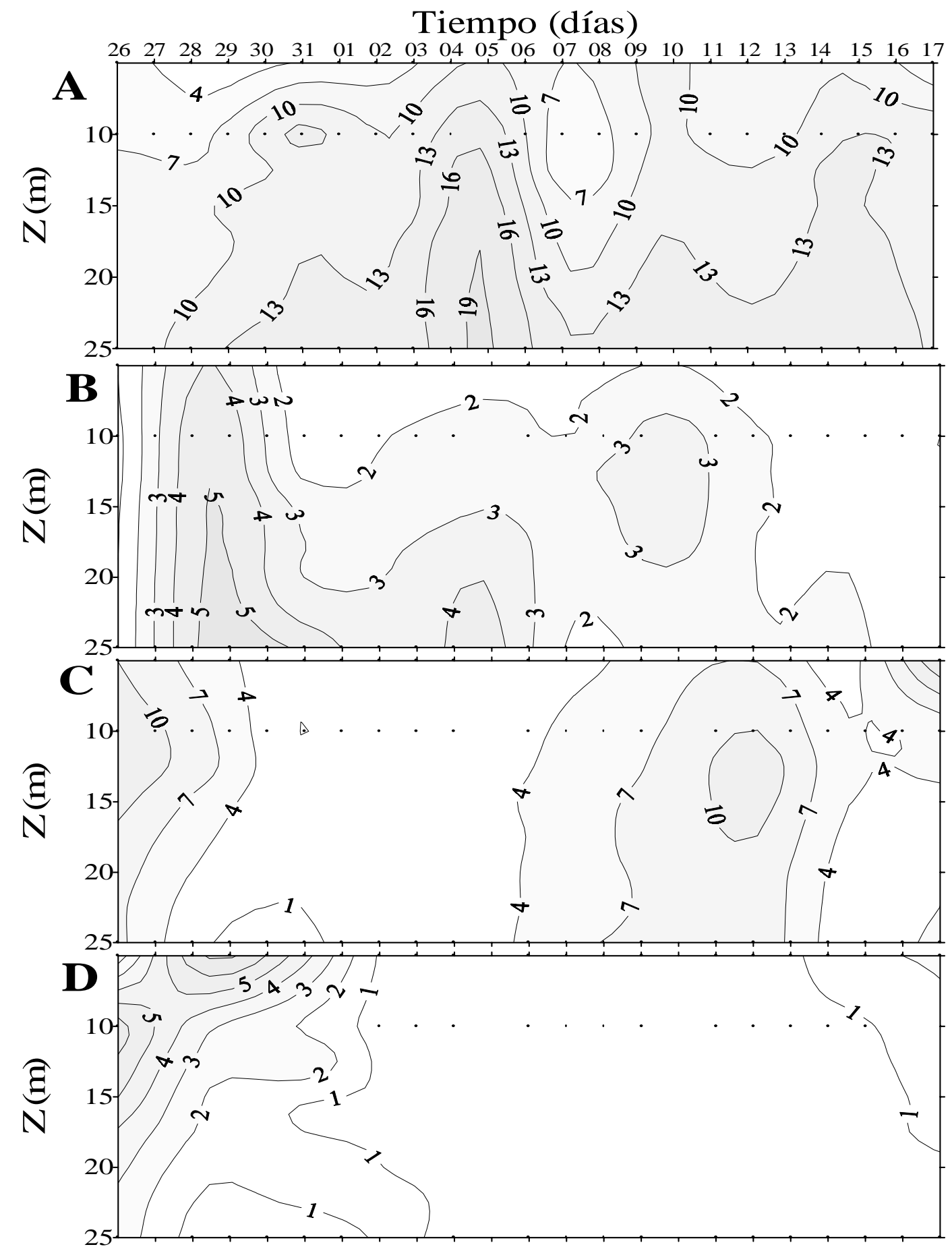

Figura 5

Distribución vertical para la estación 3 de (A) nitrato ( $\mu$ g-at N-NO ${ }_{3}$ ), (B) fosfato ( $\mu$ g-at P-PO P $_{4},(\mathrm{C})$ clorofila-a $(\mu \mathrm{g} / \mathrm{L}), \mathrm{y}$ (D) densidad celular (cél/L).

Vertical distribution for the station 3 of (A) nitrate (mg-at N-NO3), (B) phosphate (mg-at P-PO4), (C) chlorophyll-a (mg/L), and (D) cellular density (cél/L). 

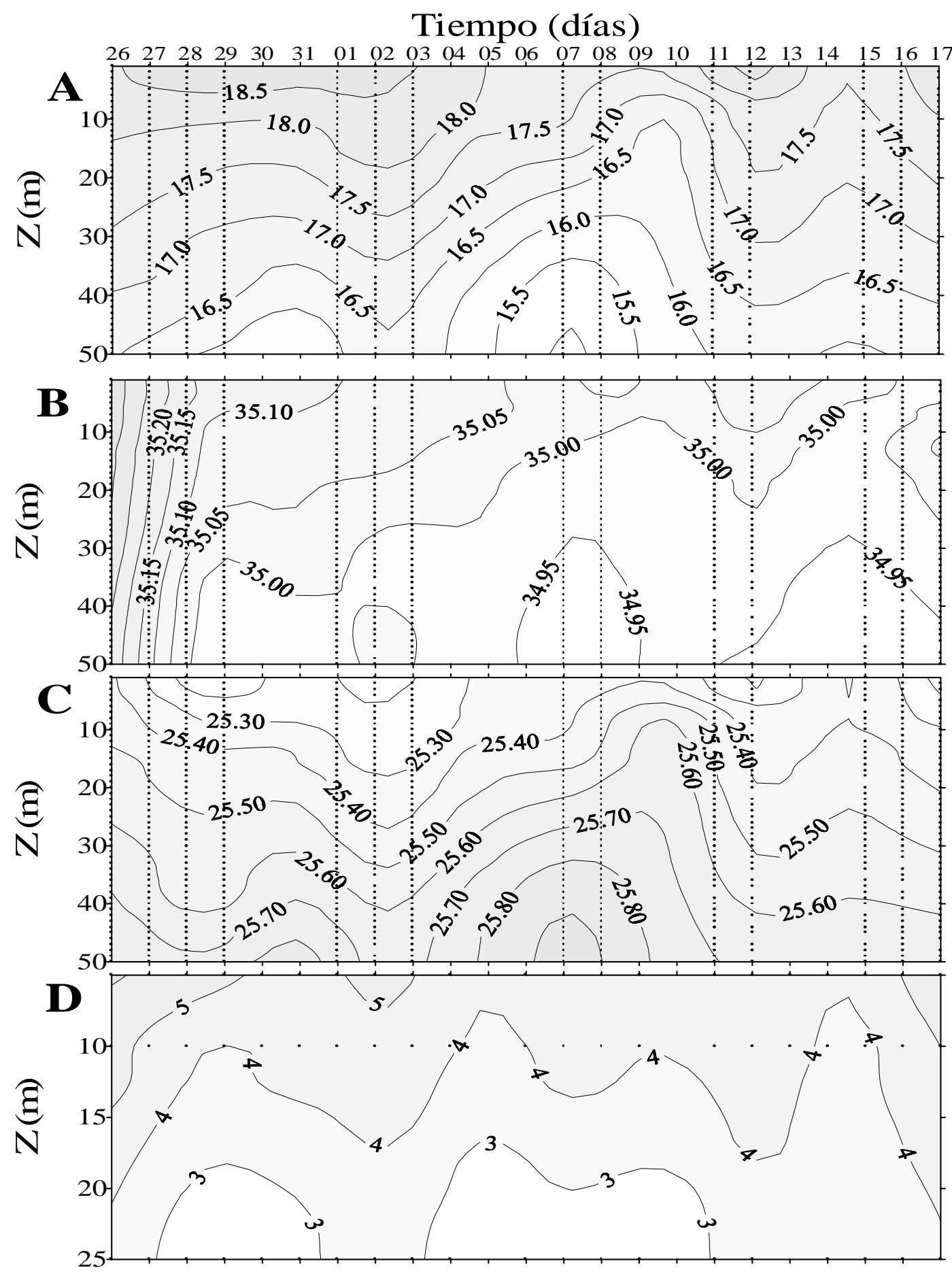

Figura 6

Distribución vertical para la estación 4 de (A) temperatura $\left({ }^{\circ} \mathrm{C}\right)$, (B) salinidad (psu), (C) densidad (unidades de sigmat), y (D) oxígeno disuelto $(\mathrm{mL} / \mathrm{L})$.

Vertical distribution for the station 4 of (A) temperature $\left({ }^{\circ} \mathrm{C}\right)$, (B) salinity (psu), (C) density (sigma-t units), and (D) dissolved oxygen $(\mathrm{mL} / \mathrm{L})$. 


\section{Tiempo (días)}
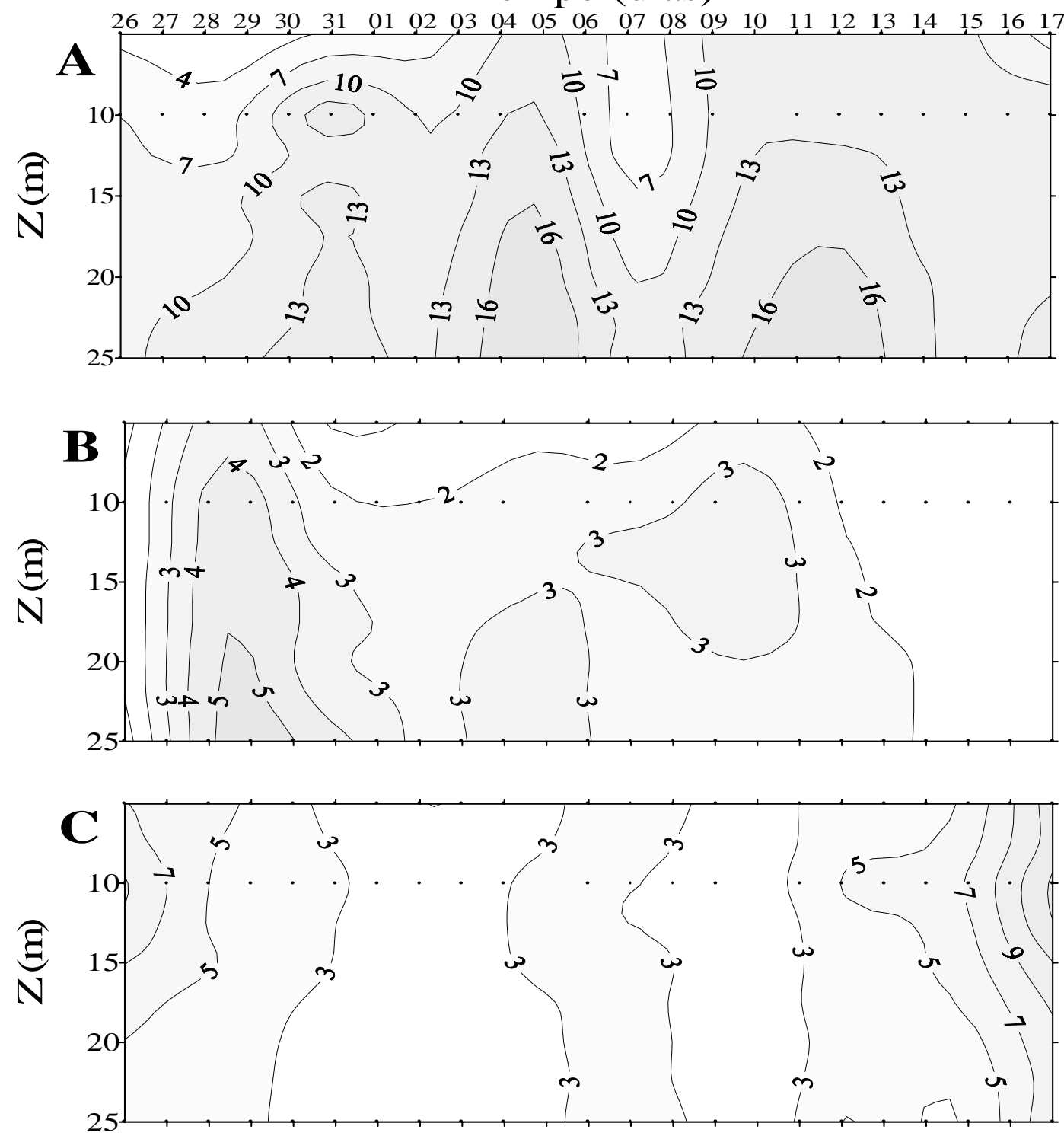

Figura 7

Distribución vertical para la estación 4 de (A) nitrato ( $\mu$ g-at $\mathrm{N}-\mathrm{NO}_{3}$ ), (B) fosfato ( $\mu$ g-at $\left.\mathrm{P}-\mathrm{PO}_{4}\right),(\mathrm{C})$ clorofila-a $(\mu \mathrm{g} / \mathrm{L})$, y (D) densidad celular (cél/L).

Vertical distribution for the station 4 of (A) nitrate (mg-at N-NO3), (B) phosphate (mg-at P-PO4), (C) chlorophyll-to (mg/L), and (D) cellular density (cél/L). 


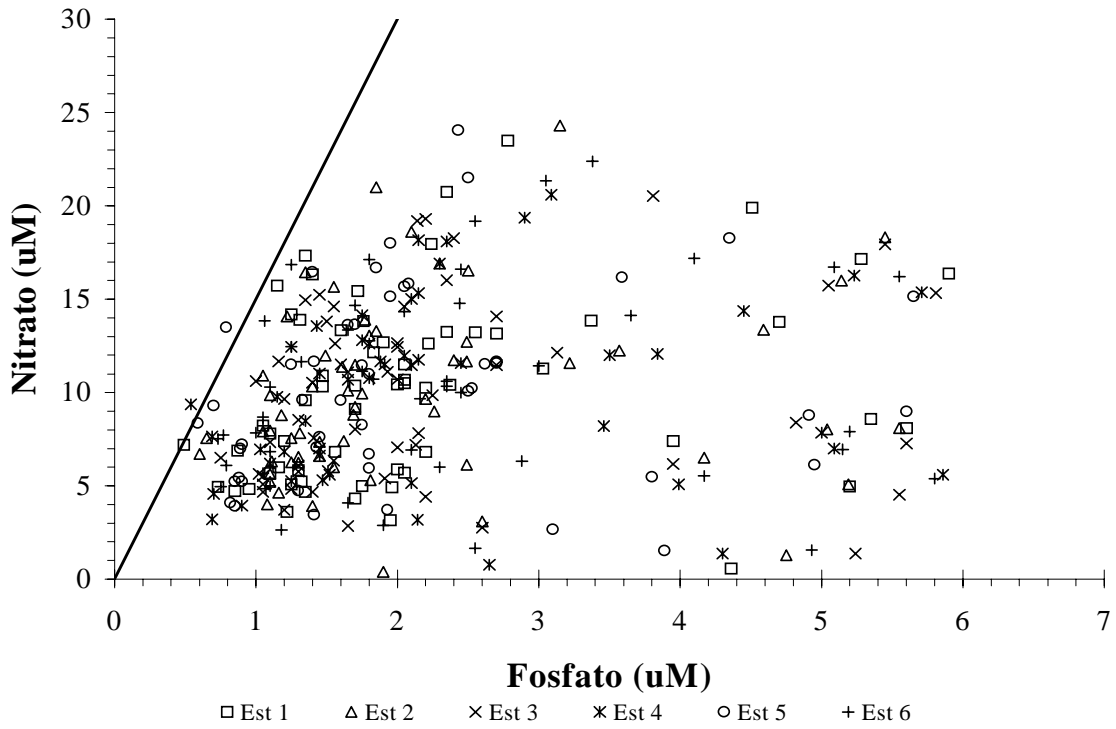

Figura 8

Relación entre la concentración de fosfato y nitrato para observaciones realizadas en el nivel de 25 m de profundidad. Donde: A, primer período; B, segundo período. Mayores explicaciones en el texto.

Relationship between the phosphate concentration and nitrate for observations carried out in the deep of $25 \mathrm{~m}$. Where: A, first period; B, second period. See explanations in the text.

Tabla 1

Resultados del análisis de correlaciones de rangos de Spearman (Zar 1984) para datos registrado en la estación 3. Los valores marcados en negrillas indican correlaciones significativas $(\mathbf{p}<0,05 ; n=17)$.

Results of the analysis of Spearman correlations coeficients (Zar 1984) for data registered in the station 3 . The values marked in bold significant correlations $(\mathrm{p}<0,05 ; \mathrm{n}=17)$.

\begin{tabular}{|c|c|c|c|c|c|c|c|c|c|c|c|}
\hline & Sal & Den & $\mathrm{N}(0-25)$ & PFZ & $\mathrm{O}_{2}$ & $\mathrm{NO}_{2}$ & $\mathrm{NO}_{3}$ & $\mathbf{P O}_{4}$ & Fito & Zoo & Cla \\
\hline Temp. & 0.679 & -0.755 & 0.167 & 0.104 & 0.370 & -0.176 & \begin{tabular}{|c|}
-0.495 \\
\end{tabular} & -0.272 & 0.216 & 0.039 & 0.309 \\
\hline $\mathrm{P}$ & 0.003 & 0.000 & 0.523 & 0.691 & 0.144 & 0.498 & 0.043 & 0.291 & 0.406 & 0.881 & 0.228 \\
\hline Sal & & -0.147 & 0.449 & 0.236 & 0.490 & 0.235 & -0.365 & -0.071 & 0.495 & 0.002 & 0.059 \\
\hline $\mathrm{P}$ & & 0.573 & 0.071 & 0.363 & 0.046 & 0.363 & 0.149 & 0.786 & 0.043 & 0.993 & 0.823 \\
\hline Den & & & 0.167 & -0.055 & -0.086 & 0.282 & 0.250 & 0.027 & 0.103 & -0.181 & $\begin{array}{l}-0.039 \\
\end{array}$ \\
\hline $\mathrm{P}$ & & & 0.523 & 0.835 & 0.743 & 0.273 & 0.333 & 0.918 & 0.694 & 0.486 & 0.881 \\
\hline $\mathrm{N}(0-25)$ & & & & -0.619 & 0.267 & 0.230 & \begin{tabular}{|c|}
-0.343 \\
\end{tabular} & 0.238 & 0.446 & 0.243 & 0.184 \\
\hline $\mathrm{P}$ & & & & 0.008 & 0.300 & 0.374 & 0.178 & 0.358 & 0.073 & 0.348 & 0.480 \\
\hline PFZ & & & & & 0.026 & 0.072 & 0.144 & -0.206 & 0.159 & \begin{tabular}{|c|}
-0.265 \\
\end{tabular} & $\begin{array}{l}-0.435 \\
\end{array}$ \\
\hline $\mathrm{P}$ & & & & & 0.921 & 0.784 & 0.582 & 0.428 & 0.543 & 0.303 & 0.081 \\
\hline $\mathrm{O}_{2}$ & & & & & & -0.037 & -0.480 & -0.176 & 0.373 & -0.076 & 0.306 \\
\hline $\mathrm{P}$ & & & & & & 0.889 & 0.051 & 0.498 & 0.141 & 0.772 & 0.232 \\
\hline $\mathrm{NO}_{2}$ & & & & & & & 0.118 & 0.537 & 0.113 & -0.007 & -0.292 \\
\hline $\mathrm{P}$ & & & & & & & 0.653 & 0.026 & 0.667 & 0.978 & 0.256 \\
\hline $\mathrm{NO}_{3}$ & & & & & & & & 0.257 & -0.353 & -0.125 & -0.326 \\
\hline $\mathrm{P}$ & & & & & & & & 0.319 & 0.165 & 0.633 & 0.202 \\
\hline $\mathrm{PO}_{4}$ & & & & & & & & & -0.110 & 0.404 & -0.365 \\
\hline $\mathrm{P}$ & & & & & & & & & 0.673 & 0.107 & 0.149 \\
\hline Fito & & & & & & & & & & 0.103 & -0.162 \\
\hline $\mathrm{P}$ & & & & & & & & & & 0.694 & 0.535 \\
\hline Zoo & & & & & & & & & & & -0.186 \\
\hline $\mathrm{P}$ & & & & & & & & & & & 0.47 \\
\hline
\end{tabular}


Tabla 2

Resultados del análisis de correlaciones de rangos de Spearman (Zar 1984) para datos registrado en la estación 4. Los valores marcados en negrillas indican correlaciones significativas $(p<0,05 ; n=15)$.

Results of the analysis of Spearman correlations coeficients (Zar 1984) for data registered in the station 4 . The values marked in bold indicate significant correlations $(\mathrm{p}<0,05 ; \mathrm{n}=15)$.

\begin{tabular}{|c|c|c|c|c|c|c|c|c|c|c|}
\hline & Sal & Den & $\mathrm{N}(0-25)$ & PFZ & $\mathbf{O}_{2}$ & $\mathrm{NO}_{2}$ & $\mathrm{NO}_{3}$ & $\mathrm{PO}_{4}$ & Zoo & Cla \\
\hline Temp & 0.818 & -0.757 & -0.175 & -0.233 & 0.821 & 0.096 & -0.386 & -0.561 & 0.582 & 0.282 \\
\hline $\mathrm{P}$ & 0.000 & 0.001 & 0.533 & 0.404 & 0.000 & 0.732 & 0.156 & 0.030 & 0.023 & 0.308 \\
\hline Sal & & -0.386 & 0.189 & -0.427 & 0.521 & 0.093 & -0.611 & -0.311 & 0.457 & 0.132 \\
\hline $\mathrm{P}$ & & 0.156 & 0.499 & 0.112 & 0.046 & 0.742 & 0.016 & 0.260 & 0.087 & 0.639 \\
\hline Den & & & 0.243 & -0.191 & -0.618 & -0.018 & -0.046 & 0.307 & -0.582 & -0.232 \\
\hline $\mathrm{P}$ & & & 0.383 & 0.495 & 0.014 & 0.950 & 0.869 & 0.265 & 0.023 & 0.405 \\
\hline $\mathrm{N}(0-25)$ & & & & -0.516 & -0.404 & -0.111 & -0.393 & 0.504 & -0.150 & -0.311 \\
\hline $\mathrm{P}$ & & & & 0.049 & 0.136 & 0.694 & 0.147 & 0.056 & 0.594 & 0.260 \\
\hline PFZ & & & & & -0.193 & -0.159 & 0.705 & 0.215 & 0.108 & -0.243 \\
\hline $\mathrm{P}$ & & & & & 0.491 & 0.572 & 0.003 & 0.442 & 0.701 & 0.382 \\
\hline $\mathrm{O}_{2}$ & & & & & & 0.079 & -0.343 & -0.789 & 0.546 & 0.496 \\
\hline $\mathrm{P}$ & & & & & & 0.781 & 0.211 & 0.000 & 0.035 & 0.060 \\
\hline $\mathrm{NO}_{2}$ & & & & & & & 0.261 & 0.157 & -0.561 & 0.382 \\
\hline $\mathrm{P}$ & & & & & & & 0.348 & 0.576 & 0.030 & 0.160 \\
\hline $\mathrm{NO}_{3}$ & & & & & & & & 0.293 & -0.389 & 0.004 \\
\hline $\mathrm{P}$ & & & & & & & & 0.289 & 0.152 & 0.990 \\
\hline $\mathrm{PO}_{4}$ & & & & & & & & & -0.493 & -0.314 \\
\hline $\mathrm{P}$ & & & & & & & & & 0.062 & 0.254 \\
\hline Zoo & & & & & & & & & & 0.139 \\
\hline & & & & & & & & & & 0.621 \\
\hline
\end{tabular}

\section{Discusión y Conclusiones}

Las condiciones durante el período de estudio claramente estuvieron moduladas por procesos de surgencia, a pesar de que correspondió a un año bajo régimen ENOS (como se discutirá posteriormente). En términos ambientales, se observó un mayor número de relaciones significativas entre estaciones de una misma línea longitudinal. La concentración de clorofila no presentó correlaciones significativas con las demás variables, lo cual se debería principalmente a la existencia de un desfase en la respuesta del fitoplancton a cambios tanto en el ambiente químico como físico, como ha sido señalado por SteemanNielsen \& Georgensen (1968). Al respecto, Ahumada (1989) señala que la existencia de un período de retardo está asociado al tiempo que necesitan para que se produzca el consumo de los nutrientes transportados por las aguas recientemente afloradas.

De igual forma, resulta interesante destacar lo observado en la progresión de clorofila-a y su relación con el número de células fitoplanctónicas, donde el máximo de clorofila no presentó concordancia con la distribución del número de células. Esta situación nos permite sugerir que en determinados momentos en el sistema existiría un predominio de células correspondientes a espectros más pequeños de tamaño (e. g., nanoplancton fotosintetizador).

Por otra parte, las condiciones oceanográficas, en fase con los eventos de viento, revelaron el ascenso de aguas subsuperficiales coincidentes con la mayor o menor intensificación de la surgencia, lo cual puede ser claramente visualizado por la estructura termal (Fig. 3 y 5). Tal situación también fue observada en el ambiente químico, ejemplificado en las concentraciones de oxígeno disuelto y nitrato. No obstante, la salinidad fue la variable que mejor representó la transformación de la capa superficial, ya que los cambios en sus condiciones se visualizan como un continuo el cual progresivamente modifica la capa superficial. En cambio, el fosfato se caracterizó por presentar un máximo hacia el comienzo del estudio para luego ir paulatinamente declinando en sus valores. A su vez, si bien las concentraciones de estos dos elementos se encontraron en niveles altos, el análisis de la relación de Redfield mostró un déficit en la carga de nitrato, lo cual sugiere que el nitrógeno sería el limitante del crecimiento del fitoplancton, lo cual ya ha sido sugerido por diversos autores (Barber \& Smith 1981, Pilson 1985, Silva 1987). 
Sin embargo, la concentración de clorofila-a siempre se mantuvo por sobre $1 \mu \mathrm{g} / \mathrm{L}$, producto de la entrada de nutrientes en concordancia con el aumento en las condiciones que favorecen la generación de surgencia. Las concentraciones detectadas son importantes, y por tanto, potencialmente no limitada por los nutrientes lo cual es una característica de este tipo de sistemas (Barber \& Smith 1981, Arcos \& Salamanca 1984, Ahumada et al. 1991). Así mismo, se debe tener en cuenta que para la zona bajo estudio los mecanismos de formación de nitrógeno y fósforo podrían ser diferentes, tal como ha sido demostrado para otras regiones (Pilson 1985).

Como se señalo anteriormente, este estudio se realizó durante un año que se encontró bajo la influencia "ENOS" (i. e., El Niño 1997-98). En tal sentido, Fuenzalida et al. (1999) señalaron que a partir de mayo de 1997 se presentaron anomalías térmicas en la región, hasta alcanzar en agosto una anomalía de $3,6^{\circ} \mathrm{C}$, lo cual fue indicativo de una clara manifestación del evento. Bajo este régimen, los eventos de surgencia mostraron una serie de anomalías asociadas al calentamiento, evidenciado por un debilitamiento de la misma tanto en la fase preparatoria como madura de El Niño (Figura 24). Estos mismos autores señalaron que durante el mes de septiembre de 1997 se observó un debilitamiento en el "ENOS", lo cual coincide con la ventana temporal analizada en este estudio.

Al respecto, la información aquí analizada muestra claramente que la surgencia como proceso no se encontró debilitada, detectándose eventos de magnitud moderada a fuerte, concordante con lo señalado por Fuenzalida (1990 y 1992). Esta condición se vio reflejada claramente en la estructura termal y halina imperantes. Así, a pesar que las condiciones ambientales del sistema se encontraban forzadas por un evento "ENOS" completamente desarrollado, durante la mitad de agosto y todo septiembre las condiciones atmosféricas y oceanográficas estuvieron más cercanas a una condición normal. Lo anterior nos permite concluir que durante el período en que se realizó este estudio los procesos de surgencia estuvieron activos, no existiendo una fase de relajación entre los eventos. Tal situación no resulta ser un hecho aislado, ya que Escribano (1998) describió una situación muy similar para la región de Antofagasta durante el año 1997.

Nuestros datos muestran claramente procesos de surgencia activos, sin embargo, llama la atención el calentamiento ocurrido entre los días 12 y 14 de septiembre de 1997. Tal situación respondería a una condición normal para la región, la cual deriva de la acción de ondas no forzadas atrapadas a la costa originadas en la región ecuatorial. Estas perturbaciones se propagan de norte a sur, y tendrían un efecto en la capa superficial, afectando la estructura térmica y la distribución de masas en las regiones costeras (Pizarro et al. 1994).

Finalmente, se puede concluir que los datos obtenidos en esta investigación indican la existencia de una alta variabilidad tanto espacial como temporal en el "standing stock" del fitoplancton; la existencia de una fuerte acoplo entre el ambiente físico y químico, con un desfase de dos a tres días en su repuesta y, la ocurrencia de una biomasa importante de fracciones pequeñas dentro de los productores primarios de la región (i. e., nanoplancton). Destacando que todo lo anterior se encuentra íntimamente relacionado a procesos de surgencia que modulan las condiciones oceanográficas del sistema, así como determinan la conducta de los fitoplancteres en escalas de corto término, lo que concuerda con otros autores (Bernal et al. 1983, Bernal 1990, Castillo et al. 1991).

\section{Agradecimientos}

Los autores agradecen el financiamiento otorgado por la Dirección de Investigaciones de la Universidad Arturo Prat. Además desean expresar sus agradecimientos a José Pineda $H$. por las facilidades dadas en el análisis de muestras y disponibilidad de espacio en su laboratorio, y a Rosalino Fuenzalida F. por su ayuda en el análisis de la información física y comentarios en el análisis de información.

\section{Literatura Citada}

Ahumada R. 1989. Producción y destino de la biomasa fitoplanctónica en un sistema de bahías en Chile central:Una hipótesis. Biología Pesquera 18: 53-66.

Ahumada R, P Matrai \& N Silva. 1991. Phytoplankton biomass distribution and relationship to nutrient enrichment during an upwelling event off Concepcion Bay Chile. Bol. Soc. Biol. Concepción, Chile. Tomo 62: 7-19.

Alvial A \& S Avaria. 1982. Proliferación de primavera del fitoplancton en la bahía de Valparaíso. II. Dinámica de las comunidades. Rev. Biol. Mar., Valparaíso, 18(1): 1-56.

Arcos D \& M Salamanca. 1984. Distribución de clorofila y condiciones oceanográficas frente a Chile Central (Latitudes $32^{\circ} \mathrm{S}-38^{\circ} \mathrm{S}$, Febrero 1982). Biología Pesquera 13: 5-14.

Arcos D, S Nuñez, L Castro \& N Navarro. 1987. Variabilidad vertical de clorofila-a en un área de surgencia frente a Chile Central. Invest. Pesq. (Chile) 34: 47-55.

Barber R \& R Smith. 1981. Coastal upwelling ecosystems. En: Analyisis of marine ecosystems. Ed. by R. Longhurst. Academid Press. 31-68.

Bakun A. 1973. Coastal upwelling indices, West Coast of North America, 1946-71. NOAA Tech. Report. NMFSSSRF. 671: 103p. 
Bernal P. 1990. La Oceanografía del sistema de corrientes de Chile-Perú en relación a las pesquerías pelágicas: Una revisión. En: Perspectivas de la actividad pesquera en Chile. Ed. M. A. Barbieri. Escuela de Ciencias del Mar, UCV, Valparaíso: 35-48.

Bernal P, F Robles \& O Rojas. 1983. Variabilidad física y biológica de la región meridional del sistema de corrientes Chile-Perú. En: G. Sharp \& J. Csirke (Eds). Actas de consultas de expertos para examinar los cambios en la abundancia y composición por especies de recursos de peces neriticos. San Jose, Costa Rica. FAC Informes de Pesca (291) Vol 3.: 683-711.

Billington N. 1991. A Comparison of Three Methods of Measuring Phytoplankton Biomass on a Daily and Seasonal Basis. Hydrobiologia, 226: 1-15.

Boje R \& M Tomczak. 1978. Ecosystem analysis and the definition of boundaries in upwelling regions. En: Upwelling ecosystems, Boje, R. and M. Tomczek, eds., Springer Verlag: 1-9.

Carpenter J. 1965. The Chesapeake Bay Institute technique for the Winkler dissolves oxigen method. Limnol. and Oceanogr., 10: 141-143.

Castillo G, H Muñoz, H González \& P Bernal. 1991. Daily analysis of abundance and size variability of fish larvae in relation to oceanic water intrusions in coastal areas. Biología Pesquera 20: 21-35.

Escribano R. 1998. Population dynamics of Calanus chilensis in the Chilean eastern boundary Humbolt current. Fish. Oceanogr. 7:3/4, 245-251.

Fuenzalida R. 1990. Variabilidad temporal de un indice de surgencia para la zona de Iquique (Lat. $20^{\circ} \mathrm{S}$ ). Invest. Cient. y Tec., Serie: Ciencias del Mar 1: 37-47.

Fuenzalida R. 1992. Proceso de surgencia en la región norte de Chile, $20^{\circ} 30^{\prime} S-21^{\circ} 45^{\prime} S$. Invest. Cient. y Tec., Serie: Ciencias del Mar 2: 79-104.

Fuenzalida R, J Blanco, R Núñez \& S Hormazabal. 1999. Efectos oceanográficos y meteorológicos durante El Niño 1997-98, en la zona de Iquique ( $\left.20^{\circ} \mathrm{S}\right)$. Invest. Cient. y Tec., Serie: Ciencias del Mar 4: 79-104.

Harris G. 1980. The Measurement of Photosynthesis in Natural Populations of Phytoplankton. En: The Physiological Ecology of Phytoplankton. Ed. by I. Morris. University of California Press, Berkeley and Los Angeles, California. 129-187.

Haury L, J McGowan \& $P$ Wiebe. 1978. Patterns and Processes in the Time-Space Scales of Plankton Distributions. En: Spacial Pattern in Plankton Communities. Steele, J. H. (ed), Ser. IV. Plenum Press, New York and London, Vol. 3, 277-327.
Herrera L. 1990. Composición, distribución y abundancia del fitoplancton marino, y su relación con parámetros físicosquímicos, en un área comprendida entre los $20^{\circ} 30^{\prime} \mathrm{S}$ y lo $21^{\circ} 45$ 'S, frente al río Loa. Tesis para optar al titulo de Biólogo Marino. Depto. Cs. del Mar, Universidad Arturo Prat. 192 pp.

Legendre L \& S Demers. 1984. Towards Dynamics Biological Oceanography and Limnology. Can. J. Fish Aquat. Sci., Vol 41:2- 19.

Marin V \& G Olivares. 1999. Estacionalidad de la productividad primaria en Bahía Mejillones del Sur (Chile): una aproximación proceso - funcional. Revista Chilena de Historia Natural72: 629-641.

Parsons T, Y Maita \& C Lalli. 1984. A Manual of chemical and biological methods for seawater analysis. Pergamon Press Ltd. Oxford, England. 173 pp.

Pilson M. 1985. Annual cycles of nutrients and chlorophyll in Narragansett Bay, Rhode Island. J. Mar. Res., 43: 849873.

Pizarro O, S Hormazabal, A González \& E. Yañez. 1994. Variabilidad del viento, nivel del mar y temperatura en la costa norte de Chile. Invest. Mar., 22: 85-101.

Santander P. 1993. Interacción a mesoescala entre la biomasa del fitoplancton y la estructura física de un área de

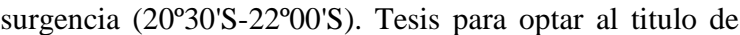
Biólogo Marino. Depto. Cs. del Mar, Universidad Arturo Prat. 80 pp.

Silva N. 1987. Contenido de sales nutrientes de las aguas del norte de Chile (Crucero MARCHILE XII-ERFEN III). Ciencia y Tecnología. del Mar, CONA, 11: 95-117.

Steeman-Nielsen E \& G Georgensen. 1968. The adaptation of plankton algae. I. General part. Physiologia plantarum, 21:401-413.

Utermöhl H. 1958. Zur vervollkommung der quantitativen phytoplankton methodik. Mitt. Int. Ver. Limnol., 9:1-38.

Wiens JA. 1989. Spatial Scalig in Ecology. Fuctional Ecology, 3: 385-397.

Wrobleski J \& J Richman. 1987. The non-linear response of plankton to winds mixing events - implications for survival of larval northern anchovy. J. Plankton Res. 9: 103-123.

Zar J. 1984. Biostatistical Analysis. Prentice Hall Inc., New Jersey. $718 \mathrm{p}$ 